\title{
Inflammatory Bowel Diseases 2003 - From Genetics to Biological Therapy
}

\author{
Jürgen Schölmerich \\ Department of Internal Medicine I, University Medical Center Regensburg, Regensburg, Germany
}

Although chronic inflammatory bowel diseases (IBD) have been described in the modern medical literature for more than 100 years, their etiology and pathogenesis are still not completely understood. In spite of this, prognosis has improved significantly. While in the 1950 s only about $80 \%$ of patients survived more than 10 years, in the mean time age-adapted survival has become almost or completely normal. The last decade has evidenced much progress in the field and new findings regarding genetic susceptibility, environmental influences, the role of bacteria, new diagnostic techniques and, in particular, new treatments have evolved. This issue of Digestive Diseases comprises a series of articles dedicated to the state of the art of the etiology, pathogenesis, diagnosis, clinical presentation, and treatment of IBD.

There is an abundance of evidence for a polygenic susceptibility to IBD. Most recently, the first gene where a mutation leads to manifestation of Crohn's disease was described, and several other candidates are being discussed. The most recent developments and the limitations of the information on the genetic basis of IBD are also discussed.

The fact that a number of temporal and geographical trends and distributions point to risk factors associated with defined life-styles has led to the suspicion that environmental factors play an important role in disease manifestation and, in addition, regarding the course and prognosis. Numerous studies have been performed on factors such as diet, smoking and infectious agents. Lately, the 'hygiene hypothesis' which indicates that childhood exposure to a high hygienic level increases the risk of developing Crohn's disease later in life has gained wide acceptance. Smoking has been found to increase the risk of Crohn's disease and decrease that of ulcerative colitis. Furthermore, appendectomy seems to decrease the risk of developing ulcerative colitis later in life. All these genetic and environmental effects have not yet been set up completely into a defined picture, but the mosaic seems to be gaining pieces. Both data from genetic and environmental studies point to the role played by bacterial flora in the manifestation and perpetuation of intestinal inflammation. Numerous animal experiments have proven that the presence of intestinal bacteria seems to be a necessary prerequisite for the manifestation of inflammation in almost all models of IBD. Furthermore, the effects of antibiotics and probiotics in experimental models and in some aspects of clinical IBD support an important role of bacteria in the pathophysiology of IBD.

During the last decade, imaging procedures such as computed tomography and magnetic resonance imaging have provided us with amazing possibilities. In addition, capsule endoscopy has opened up avenues enabling visualization of the small bowel way beyond earlier expectations. Although it is not yet clear to what extent conventional radiological techniques or even endoscopy will be substituted by these new modalities, it is obvious at the moment that small bowel imaging will probably be done using magnetic resonance imaging and/or capsule endos-

\begin{tabular}{ll}
\hline KARGER & ( ) 2003 S. Karger AG, Basel \\
0257-2753/03/0212-0083\$19.50/0 \\
$\begin{array}{l}\text { Fax +41 61306 1234 } \\
\text { E-Mail karger@karger.ch } \\
\text { www.karger.com }\end{array}$ & $\begin{array}{l}\text { Accessible online at: } \\
\text { www.karger.com/ddi }\end{array}$
\end{tabular}

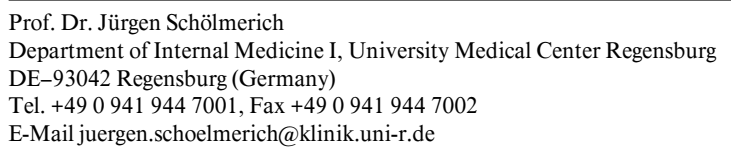


copy. In contrast, the large bowel will probably remain the domain of endoscopy as biopsy taking is only possible with real endoscopy. It is not clear at the moment to which extent magnetic resonance imaging will be able to replace other techniques regarding the assessment of disease activity.

There are a number of extraintestinal symptoms and complications in IBD. Among them, osteoporosis, which can be due to the disease itself or to treatment, remains prominent. There is still no consensus on the treatment of osteoporosis in patients with IBD and there is a lack of sufficient knowledge regarding the treatment of some extraintestinal manifestations. In contrast, extraintestinal complications which are due to the lack of gut function can be detected and treated accordingly when the pathophysiological principles are known to the patients or physicians.

Standard treatment of Crohn's disease and ulcerative colitis is relatively uniform and agreed upon worldwide. It has been tested for safety and efficacy in controlled clinical trials. However, there is the need and opportunity to adapt therapies to individual cases based on scientific facts and recent thinking, weighing up the potential effects against any side effects. Treatment concepts regarding active disease and maintenance of remission have been agreed upon in consensus guidelines; 5-aminosalicylic acid, corticosteroids, immunosuppressants, probiotics and antibiotics and Infliximab, an anti-TNF antibody, play a role.

In spite of the success of these conventional treatments, surgical therapy is still required in a large proportion of patients with ulcerative colitis and Crohn's disease at some time during their life. The need for surgery should probably not be perceived as a failure of medical therapy but rather be viewed as a reasonable part of the therapeutic arsenal for those disorders. Improvements in surgical therapies have certainly added to the normalization of lifetime prognosis in both patient populations.

'Biological therapies' based on new knowledge of pathophysiology are being tested worldwide to a surprising extent. Only infliximab, a chimeric antibody to tumor necrosis factor- $\alpha$, has been introduced into the routine arsenal. Many of the approaches have not been found to be clinically relevantly effective although they show 'biological activity'. This may be due to the fact that the arsenal of inflammatory cells and mediators is comparable to an orchestra which will not be silenced by eliminating a single instrument. The conductor of the orchestra has not yet been found and it seems doubtful that it will ever be when we consider different genotypes and consequently phenotypes of those disorders which are the basis of different effects of drugs.

In summary, there is much progress regarding inflammatory bowel disease. We still need to define more of the genotypes which will be much easier due to the knowledge of the human genome. We will then have to define treatments for the different genotypes/phenotypes and thus improve the quality of life of our patients which nowadays have a normal life expectancy. It is to be hoped that this issue of Digestive Diseases provides the necessary information for clinicians and researchers to advance our knowledge on IBD and its treatment possibilities. 